\title{
POLISH GROUP ACTIONS AND THE VAUGHT CONJECTURE
}

\author{
RAMEZ L. SAMI
}

\begin{abstract}
We consider the topological Vaught conjecture: If a Polish group $G$ acts continuously on a Polish space $S$, then $S$ has either countably many or perfectly many orbits. We show

1. The conjecture is true for Abelian groups.

2. The conjecture is true whenever $G, S$ are recursively presented, the action of $G$ is recursive and, for $x \in S$ the orbit of $x$ is of Borel multiplicative rank $\leq \omega_{1}^{x}$.

Assertion 1 holds also for analytic $S$. Specializing $G$ to a closed subgroup of $\omega$ !, we prove that nonempty invariant Borel sets, not having perfectly many orbits, have orbits of about the same Borel rank. An upper bound is derived for the Borel rank of orbits when the set of orbits is finite.
\end{abstract}

\section{INTRODUCTION}

The Vaught conjecture in model theory suggests that a first-order theory $T$ (in a countable language) has either countably many or else continuum many isomorphism types of countable models. A natural extension of the Vaught conjecture to descriptive set theory is the following:

Topological Vaught conjecture (T.V.C). If a Polish topological group $G$ acts continuously on a Polish space $S$ then either the number of orbits is countable or else there is a perfect ${ }^{1}$ set $P \subseteq S$ any two distinct elements of which lie in distinct orbits.

(This was actually first conjectured by D. E. Miller, see [25, Appendix].)

It is our aim here to prove, inter alia, two instances of the conjection: the first instance is Theorem 2.1 in $\S 2$ where the T.V.C. is established for Abelian Polish groups. The second instance is Theorem 3.8 of $\S 3$ where the conjecture is shown to hold whenever the space $S$ is recursively presented and for any $x \in S$ the orbit of $x$ is $\Pi_{\omega_{1}^{x}}^{0}$. (The relevance of this assumption is discussed in $\S \S 3$ and 5.) As stated without proof in $\S 3$, Theorem 3.8 can be improved when $G$ is a closed subgroup of $\omega$ ! (the permutation group of the natural numbers). While the first mentioned result $(2.1)$ has no immediate bearing on model theory, the

Received by the editors April 1, 1988 and, in revised form, October 23, 1989.

1980 Mathematics Subject Classification (1985 Revision). Primary 03E15, 03C15, 54H15; Secondary $04 \mathrm{~A} 15,54 \mathrm{H} 05$.

The author acknowledges with pleasure that this work was completed while he held a visiting appointment at the University of Paris VII. Special thanks are due to the Logic Group.

${ }^{1}$ Throughout, perfect sets are assumed to be nonempty, by definition. 
second (3.8) clearly has. This last result, as well as Theorem 3.2, where we compute the Borel rank of the orbit of $x$, extend to the wider context of group actions well-known model-theoretic results of Makkaï and of Nadel.

In $\S 4$ we restrict attention to groups $G$ which are closed subgroups of $\omega$ !. Our main result there is Theorem 4.6, which gives a sharp bound on the Borel rank of the orbits if the number of orbits is finite. This is only indirectly connected to the T.V.C. but is derived from a quite general basis theorem for invariant Borel subsets without a perfect set of orbits: Theorem 4.5. Iil $\S 5$ connections between our results and model theoretic results are briefly discussed. Weaker versions of Theorems 3.8, 4.5, and 4.6 were announced and sketched in [28].

The work presented here is in line with the program implicit in Vaught's [35]: use descriptive set-theoretic methods to shed light on model theoretic problems. A debt of inspiration is also owed to work of Miller [17], and Burgess and Miller [5]. I wish to thank D. E. Miller, and A. Louveau and J. Stern for inspiring conversations on the matter at hand.

\section{Preliminaries AND background}

1.1. Let $L$ be a countable first-order language, $L_{\omega_{1} \omega}$ denotes as usual the infinitary language with countable conjunctions and disjunctions (see [1, Chapter III]). A subset $F \subseteq L_{\omega_{1} \omega}$ is called a fragment if it is closed under substitution of variables and satisfies: (i) $F$ contains the atomic formulas, (ii) $F$ is closed under subformulas, and (iii) $F$ is closed under negation. (This notion of fragment is quite a bit more general than the usual one, cf. [1].)

The set of $L$-structures with universe $\omega$ will be denoted $X_{L}$. For $\varphi(\vec{x}) \in$ $L_{\omega_{1} \omega}$ and $\vec{s}$ a finite sequence from $\omega$ of the appropriate length, let

$$
\operatorname{Mod}(\varphi, \vec{s})=\left\{\mathscr{M} \in X_{L} \mid \mathscr{M} \models \varphi[\vec{s}]\right\} .
$$

Given a fragment $F \subseteq L_{\omega_{1} \omega}$, a topology $\mathscr{T}_{F}$ is generated on $X_{L}$ by the sets $\operatorname{Mod}(\varphi, \vec{s})$, for $\varphi \in \bar{F}, \vec{s} \in \omega^{<\omega}$. Let $\mathscr{Z}_{L, F}$ denote $X_{L}$ equipped with the topology $\mathscr{T}_{F}$. The following is essentially in [10] (see also 4.2 below, for a proof).

Proposition. Let $F \subseteq L_{\omega_{1} \omega}$ be a countable fragment. The topological space $\mathscr{Z}_{L, F}$ is a Polish space, and the map

$$
\begin{aligned}
J: \omega ! \times X_{L} & \rightarrow X_{L} \\
\langle f, \mathscr{M}\rangle & \mapsto f \cdot \mathscr{M}
\end{aligned}
$$

is a continuous group action of the Polish group $\omega$ ! on $\mathscr{X}_{L, F}$. (Here $f \cdot \mathscr{M}$ is just the isomorphic copy of $\mathscr{M}$ under the permutation $f$.)

This proposition justifies our initial statement that the Vaught conjecture follows from the T.V.C. Indeed, a stronger version about the number of models of a sentence of $L_{\omega_{1} \omega}$ does follow. Given a sentence $\sigma \in L_{\omega_{1} \omega}$, let $F$ be a countable fragment with $\sigma \in F$ and set $S=\operatorname{Mod}(\sigma, \varnothing) . S$ is a clopen set in $\mathscr{X}_{L, F}$; thus, with the induced topology, $S$ is Polish. Looking at the induced action $J^{\prime}: \omega ! \times S \rightarrow S$ one sees that counting the isomorphism types of countable models of $\sigma$, amounts to counting the orbits in $S$.

1.2. It was shown by Kunen that the Vaught conjecture does not hold for pseudo-elementary classes (see [31] for a proof). In topological terms this means 
that the strengthening of the T.V.C. to actions $J: G \times S \rightarrow S$ where $S$ is analytic is false in general.

However, nothing presently known precludes the truth of the conjecture when one relaxes the conditions to (i) the space $S$ is Lusin (i.e., a Borel subset of a Polish space), and (ii) $J$ is Borel measurable. Indeed this strengthened conjecture seems as plausible as the T.V.C. (It is an easy consequence of the main result of [6] that if $J: G \times S \rightarrow S$ is separately continuous, where $G$ is Baire and $S$ is metrizable separable, then $J$ is actually continuous. Thus separate continuity need not be considered here.)

1.3. From now on, and for the duration of the paper, $G$ is a Polish group, $S$ a metrizable space and $J: G \times S \rightarrow S$ is a Borel measu:able group action. As for notation, $f \cdot x$ denotes $J(f, x), \mathscr{E}_{J}$ (or just $\mathscr{E}$ ) denotes the equivalence relation induced on $S$, and $[x]$ denotes the orbit (E्E-equivalence class) of $x$.

Miller, in [16, Theorem 2], has shown that the orbits of $\mathscr{E}_{J}$ are Borel. (RyllNardjewski [26] and Dixmier [8] had already shown this for the case where $S$ is Polish and $J$ is continuous.)

A very useful tool here consists of the Vaught transforms. If $B \subseteq S$, and $U \subseteq G$ is open, one defines the transforms

$B^{\Delta U}=\{x \in S \mid\{f \in G \mid f \cdot x \in B\}$ is nonmeager in $U\}$,

$B^{* U}=\sim\left((\sim B)^{\Delta U}\right)$;

also let, $B^{\Delta}=B^{\Delta G}$ and $B^{*}=B^{* G}$.

We collect here, for convenience, the Vaught results [35] that are needed. $1.4(\mathrm{v})$ is due to Miller [18, 3.1].

1.4. Theorem. (i) $B^{*} \subseteq B^{\Delta}$, where both are invariant subsets of $S$. If $B$ is invariant then

$$
B=B^{*}=B^{\Delta} \text {. }
$$

(ii) $\left(\bigcup_{i<\omega} B_{i}\right)^{\Delta U}=\bigcup_{i<\omega} B_{i}^{\Delta U}$ and $\left(\bigcap_{i<\omega} B_{i}\right)^{* U}=\bigcap_{i<\omega} B_{i}^{* U}$.

(iii) If $\mathscr{B}$ is any countable basis for $G$, and the sets $\{f \in G \mid f \cdot x \in B\}$ have the Baire property for all $x \in S$, then

$$
B^{\Delta U}=\bigcup\left\{B^{* V} \mid V \subseteq U, V \in \mathscr{B}\right\}, \quad B^{* V}=\bigcap\left\{B^{\Delta V} \mid V \subseteq U, V \in \mathscr{B}\right\} .
$$

(iv) Suppose $J$ is continuous. For all countable ordinals $\xi \geq 1$,

$$
\begin{aligned}
& A \subseteq S \text { is } \boldsymbol{\Sigma}_{\xi}^{0} \Rightarrow A^{\Delta U} \text { is } \boldsymbol{\Sigma}_{\xi}^{0}, \\
& A \subseteq S \text { is } \boldsymbol{\Pi}_{\xi}^{0} \Rightarrow A^{* U} \text { is } \boldsymbol{\Pi}_{\xi}^{0} .
\end{aligned}
$$

(v) If $J$, instead, is $\Sigma_{1+\mu}^{0}$-measurable, where $\mu<\omega_{1}$, then

$$
\begin{aligned}
& A \subseteq S \text { is } \boldsymbol{\Sigma}_{\xi}^{0} \Rightarrow A^{\Delta U} \text { is } \boldsymbol{\Sigma}_{\mu+\xi}^{0}, \\
& A \subseteq S \text { is } \boldsymbol{\Pi}_{\xi}^{0} \Rightarrow A^{* U} \text { is } \boldsymbol{\Pi}_{\mu+\xi}^{0} .
\end{aligned}
$$

1.5. Let now $E$ be an equivalence relation on a Polish space $S$ and $Y \subseteq S$. As usual, one says that $Y$ meets perfectly many classes (or orbits) if there is a perfect subset $P \subseteq Y$ consisting of mutually $E$-inequivalent points. In this case $Y / E$ has (effectively) cardinality $2^{\aleph_{0}}$. One extends this notion to a more general metrizable space $S$, by requiring a $P$, as above, which is perfect and compact. (Observe that, in a Polish space, a perfect set contains a copy of the Cantor space.) 
1.6. The following observation will be used a couple of times. Suppose $T$ is Polish, $S$ is metrizable, $F: T \rightarrow S$ is continuous, and $Y=F[T]$. Let $E$ be an equivalence relation on $S$. Denote by $\widehat{E}$ the pullback of $E$ by $F$, namely: $x \widehat{E} y \Leftrightarrow F(x) E F(y)$. Then: If $T$ has perfectly [resp. countably] many $\widehat{E}$ classes then $Y$ meets perfectly [resp. countably] many E-classes. To prove this, let $P \subseteq T$ be a perfect set consisting of pairwise $\widehat{E}$-inequivalent points. Then one can extract a compact perfect subset $P^{\prime} \subseteq P$. The image $F\left[P^{\prime}\right]$ is compact and perfect in $S$. It witnesses that $Y$ meets perfectly many classes. The case of a countable number of classes is trivial.

1.7. A theorem we shall use repeatedly is due to Silver [30]: Assume $E$ is a $\Pi_{1}^{1}$ equivalence relation on the Polish space $S$ and $Y \subseteq S$ is $\Sigma_{1}^{1}$. Then $Y$ meets countably or perfectly many E-classes.

Silver's theorem is actually stated for $Y=S$. To get the present statement, let $F: \omega^{\omega} \rightarrow S$ be continuous with $F\left[\omega^{\omega}\right]=Y$. The pullback of $E$ by $F$ is $\Pi_{1}^{1}$. So Silver's theorem applies to $\widehat{E}$. Now use 1.6.

1.8. Given $J: G \times S \rightarrow S$ as above, if the space $S$ is analytic then $\mathscr{E}$, the induced equivalence relation, is also analytic. Thus, by the main result of Burgess [3], on $\Sigma_{1}^{1}$ equivalence relations one knows that the space $S$ has at most $\aleph_{1}$ or perfectly many $\mathscr{E}$-orbits.

Our notation and terminology for descriptive set theory follow Moschovakis [19], in particular as regards the notion of recursively presented space. For firstorder logic, Chang and Keisler [7] is a convenient reference, while Barwise's [1] is our basic reference for "effective" infinitary logic.

\section{2. i He Abelian CASE}

As usual, one defines the stabilizer subgroup of $x \in S$ to be $G_{x}=\{f \in G \mid$ $f \cdot x=x\}$.

If $J$ is continuous, $G_{x}$ is obviously closed. Miller [16] shows that this is the case even when $J$ is just Borel measurable. A theorem of Ryll-Nardjewski [26] and Dixmier [8] shows that given a closed subgroup $H \subseteq G$ there is a Borel (in fact $G_{\delta}$ ) selector $K \subseteq G$ for the partition $G / H$ of $G$ into left cosets.

2.1. Theorem. Let $J: G \times S \rightarrow S$ be a Borel measurable group action of the Polish group $G$ on the analytic space $S$. If for all $x \in S, G_{x}$ is a normal subgroup of $G$, then $S$ contains either countably many or perfectly many orbits.

2.2. Corollary. If the group $G$ is Abelian or Hamiltonian, then $S$ contains either countably many or perfectly many orbits.

Proof of the theorem. Assume first for simplicity that $S$ is Polish. Given $x, y \in$ $S$ it is easily verified that, whenever $g \cdot x=y$ then $g G_{x} g^{-1}=G_{y}$. Thus for any $x, y \in S$, since $G_{x}$ is a normal subgroup of $G$, one has $x \mathscr{E} y \Rightarrow G_{x}=G_{y}$. Let $\rho$ now denote the following equivalence relation on $S$ :

$$
x \rho y \Leftrightarrow G_{x}=G_{y} .
$$

$\rho$ is coarser than $\mathscr{E}$. Further one can write

$$
x \rho y \Leftrightarrow \forall g \in G(g \cdot x=x \Leftrightarrow g \cdot y=y) .
$$

$J$ being $\Delta_{1}^{1}, \rho$ is thus $\Pi_{1}^{1}$. One now applies Silver's theorem to $\rho$ : 
Case 1. There are perfectly many $\rho$-classes. In this case, there are a fortiori perfectly many $\mathscr{E}$-orbits.

Case 2. $S$ has countably many $\rho$-classes. We claim that $\mathscr{E}$ is $\Delta_{1}^{1}$. This claim would finish the proof as one need only apply Silver's theorem once more, this time to $\mathscr{E}$. To prove the claim, let $\left\langle C_{i} \mid i<\omega\right\rangle$ be an enumeration of the $\rho$-classes. Observe first that each $C_{i}$ is $\Delta_{1}^{1}$, being $\Pi_{1}^{1}$ and the complement of a countable union of $\Pi_{1}^{1}$ sets. Further, by definition of $\rho$, the members of a $C_{i}$ all have the same stabilizer: $H_{i}$, say.

Using now the theorem of Dixmier and Ryll-Nardjewski quoted above, let $K_{i} \subseteq G$ be a Borel selector for $G / H_{i}$ (the left coset partition). One sees that for $x, y \in C_{i}$, if $x \mathscr{E} y$ then $\{g \in G \mid g \cdot x=y\}$ is a left $H_{i}$-coset, thus for $x, y \in C_{i}$,

$$
x \mathscr{E} y \Leftrightarrow\left(\exists g \in K_{i}\right)(g \cdot x=y) \Leftrightarrow\left(\exists ! g \in K_{i}\right)(g \cdot x=y) .
$$

Thus $\mathscr{E} \mid C_{i}$ is the one-to-one projection of the Borel set $\{\langle g, x, y\rangle \mid x, y \in$ $\left.C_{i} \& g \cdot x=y\right\}$ By a classical result of Luzin [19, 2E.9], $\mathscr{E} \mid C_{i}$ is $\Delta_{1}^{1}$. But $\mathscr{E}=\bigcup_{i<\omega}\left(\mathscr{E} \mid C_{i}\right)$, thus $\mathscr{E}$ is $\Delta_{1}^{1}$ as claimed.

The general case, where $S$ is analytic, is only notationally more complex: one uses a continuous surjection $F: T \rightarrow S$ where $T$ is Polish and one essentially pulls back everything to $T$. While $\widehat{\mathscr{E}}$, the pullback of $\mathscr{E}$, does not quite arise from a group action, similar arguments apply to show that $T$ has either countably many or perfectly many $\widehat{\mathscr{E}}$-classes. Conclude by invoking 1.6.

Remark. As observed by Burgess [4], if $G$ is locally compact and $J$ is continuous then $\mathscr{E}_{j}$ is $F_{\sigma}$, thus Silver's theorem applies directly in this case to yield the desired conclusion. We do not know whether or not, in the Abelian case, $\mathscr{E}_{J}$ is Borel.

\section{RECURSIVENESS CONDITIONS}

In studying countable models, say $\langle\omega, R\rangle$, where $R \subseteq \omega \times \omega$, the fact that the usual action $\omega ! \times \mathscr{P}(\omega \times \omega) \rightarrow \mathscr{P}(\omega \times \omega)$ is recursive is used to gain information on the orbits. More generally, our results deal with $\Delta_{1}^{1}$ group actions. Moschovakis' notion of recursively presented (henceforth: r.p.) Polish space is assumed here. The advantage of this "abstract" setting is that-save for $\omega !-$ a Polish group seldom appears naturally as a subspace of $\omega^{\omega}$.

Let $X, Y$ vary over r.p. Polish spaces. For $x \in X, \omega_{1}^{x}$ is defined as usual (see [19, 4F.3]). A direct computation shows that the relation " $x \in X$, $y \in Y$, and $\omega_{1}^{x} \leq \omega_{1}^{y \prime}$ is $\Sigma_{1}^{1}$. Spector's theorem: $\omega_{1}^{x}=\operatorname{Sup}\{|u| \mid u \in W O$ and $\left.u \in \Delta_{1}^{1}(x)\right\}$ also holds (with proof identical to [19, 4A.5]). Consequently, whenever $G: X \rightarrow Y$ is a $\Delta_{1}^{1}$ isomorphism, then $\omega_{1}^{x}=\omega_{1}^{G(x)}$. This last observation, in conjunction with the following proposition [19, 3E.7]: There is a $\Delta_{1}^{1}$ isomorphism $F: X \rightarrow \omega^{\omega}$, can now be used to translate well-known results about $\omega^{\omega}$ to our setting. In particular we shall use the following results: Gandy's basis theorem [9]: Suppose $A \subseteq X$ is $\Sigma_{1}^{1}(y)$ and nonempty, $y \in Y$, there is $x \in X$ such that $\omega_{1}^{x} \leq \omega_{1}^{y}$. Sacks' theorem [27]: For $\gamma \in \omega^{\omega}$, if $\xi>\omega$ is a countable $\gamma$-admissible ordinal, there is $x \in X$ such that $\omega_{1}^{(x, \gamma)}=\xi$.

For the remainder of this section we assume the following: $G, S$ are recursively presented (perfect) Polish spaces and $J: G \times S \rightarrow S$ is a $\Delta_{1}^{1}$ group action. 
For any $x \in S$ set

$$
\omega_{1}^{[x]}=\operatorname{Inf}\left\{\omega_{1}^{y} \mid y \in[x]\right\}
$$

and for $A \subseteq S$, set

$$
\operatorname{Spec}(A)=\left\{\omega_{1}^{[x]} \mid x \in A\right\}
$$

the ordinal spectrum of $A$. Given a parameter $\gamma \in \omega^{\omega}$, these notions are relativized to $\gamma$ as follows. $\omega_{1}^{[x, \gamma]}=\operatorname{Inf}\left\{\omega_{1}^{(y, \gamma)} \mid y \in[x]\right\}$, and $\operatorname{Spec}^{\gamma}(A)=$ $\left\{\omega_{1}^{[x, y]} \mid x \in A\right\}$.

3.1. Proposition (Hinman [11]). Suppose $X, Y$ are r.p. Polish spaces. For all $y \in Y$, the set $\left\{x \in X \mid \omega_{1}^{(x, y)}=\omega_{1}^{y}\right\}$ is comeager in $X$.

Proof. Note that the complement of the set above is $C=\left\{x \mid \omega_{1}^{(x, y)}>\omega_{1}^{y}\right\}$, a $\Pi_{1}^{1}(y)$ set. If $C$ were nonmeager then, by the Sacks-Tanaka basis theorem for nonmeager $\Pi_{1}^{1}$ sets $[19,4 \mathrm{~F} .20]$, there would be $x \in C$, a $\Delta_{1}^{1}(y)$ point. But then, $\langle x, y\rangle \in \Delta_{1}^{1}(y)$ and thus $\omega_{1}^{(x, y)} \leq \omega_{1}^{v}$-a contradiction.

3.2. Lemma. Given $x \in S,\left\{g \in G \mid \omega_{1}^{g \cdot x}=\omega_{1}^{[x]}\right\}$ is comeager in $G$.

Proof. Set $\xi=\omega_{1}^{[x]}$ and pick $\bar{x} \in[x]$ with $\omega_{1}^{\bar{x}}=\xi$, say $\bar{x}=\bar{f} \cdot x$. Observe that for $g \in G, g \cdot \bar{x}=g \bar{f} \cdot x$, thus $\left\{g \mid \omega_{1}^{g \cdot x}=\xi\right\} \bar{f}^{-1}=\left\{g \mid \omega_{1}^{g \cdot \bar{x}}=\xi\right\}$. Since translation in $G$ preserves category, it suffices to check that the latter set is comeager. Note that for $g \in G, g \cdot \bar{x} \in \Delta_{1}^{1}(g, \bar{x})$, hence $\omega_{1}^{g \cdot \bar{x}} \leq \omega_{1}^{(g, \bar{x})}$. Consequently, $\left\{g \mid \omega_{1}^{g \cdot \bar{x}}=\xi\right\} \supseteq\left\{g \mid \omega_{1}^{(g, \bar{x})}=\xi\right\}$. By 3.1, this last set is comeager. Hence, the conclusion.

3.3. Theorem. (a) The relation " $x, y \in S$ and $\omega_{1}^{[x]} \leq \omega_{1}^{[y]}$ " is $\Sigma_{1}^{1}$.

(b) Given $A \subseteq S, A$ in $\Sigma_{1}^{1}(\gamma), \operatorname{Spec}^{\gamma}(A)$ is an initial segment of the $\gamma$ admissible ordinals $>\omega$.

Proof. (a) $\omega_{1}^{[x]} \leq \omega_{1}^{[y]}$ is equivalent to $\exists \bar{x}\left(\bar{x} \mathscr{C}_{J} x\right.$ and $\left.\omega_{1}^{\bar{x}} \leq \omega_{1}^{[y]}\right)$. Now the set $A=\left\{\langle g, u, v\rangle \in G \times S \times S \mid \omega_{1}^{u} \leq \omega_{1}^{g \cdot v}\right\}$ is $\Sigma_{1}^{1}$, and in view of 3.2, $\omega_{1}^{\bar{x}} \leq \omega_{1}^{[y]}$ is equivalent to: " $\{g \in G \mid A(g, \bar{x}, y)\}$ is comeager in $G$." By the category computations in [12] (see [19,4F.19]), one sees that this is a $\Sigma_{1}^{1}(\bar{x}, y)$ statement. This proves (a). (This is also close to Vaught's: if $A$ is $\Sigma_{1}^{1}$, so is $A^{*}$.)

(b) As usual, we may drop $\gamma$ from the argument. Suppose $A \neq \varnothing$ and $\xi<\operatorname{Sup}(\operatorname{Spec}(A))$ with $\xi>\omega$, admissible. By Sacks' theorem, let $\alpha \in \omega^{\omega}$ have $\omega_{1}^{\alpha}=\xi$. The set $C=\left\{x \in A \mid \omega_{1}^{\alpha} \leq \omega_{1}^{[x]}\right\}$ is nonempty. By 3.2, $x \in C$ is equivalent to " $\left\{g \in G \mid \omega_{1}^{\alpha} \leq \omega_{1}^{g * x}\right\}$ is comeager." Arguing as in (a) above, one sees that $C$ is a $\Sigma_{1}^{1}(\alpha)$ set. By the Gandy basis theorem, there is $x \in C$ with $\omega_{1}^{x} \leq \omega_{1}^{\alpha}$. But then, necessarily $\omega_{1}^{[x]}=\omega_{1}^{\alpha}=\xi$.

Remark. The model-theoretic special case of 3.3 is implicit in Ressayre's study of $\Sigma$-saturated models [24]. Our method here is, by necessity, quite different.

An important fact about a model $M$ with universe $\omega$, is that its isomorphism class is in $\Pi_{\omega_{1}^{M}+2}^{0}$ (see [31]). This uses Nadel's study of the Scott rank [21]. We can generalize this result to the topological situation by a different (and perhaps, simpler) proof. 
3.4. Lemma. Suppose $X$ is an r.p. Polish space and $A \subseteq X$ is $\Sigma_{1}^{1}$. If for all $x \in A, \omega_{1}^{x} \leq \xi$ (a countable ordinal), then $A$ is $\Pi_{\xi+2}^{0}$.

Proof. As usual, let $F: X \rightarrow \mathscr{P}(\omega \times \omega)$ be a $\Delta_{1}^{1}$ function such that for all $x \in X, F(x)$ is a linear ordering of $\omega$ and: $x \in A \Leftrightarrow F(x) \notin W O . F$ can be assumed to be $\Sigma_{2}^{0}$-measurable (see the proof of [19, 4A.3]). Now for $x \in A$, any well-ordered initial segment of $F(x)$, with a top element, is $\Delta_{1}^{1}$ in $x$. Such a segment must have length $<\omega_{1}^{x} \leq \xi$. Hence we can write: $x \in A \Leftrightarrow F(x) \in T_{\xi}$, where $T_{\xi}$ here denotes the set of linear orderings of $\omega$, which are not well founded and have largest well-ordered initial segment of length $\leq \xi$.

We now argue that $T_{\xi}$ is a $\Pi_{\xi+2}^{0}$ set. For $\nu<\omega_{1}$, let $W O^{<\nu}$ denote the set of (nonstrict) well-orderings of length $<\nu$, with field a subset of $\omega$. Given $u \subseteq \omega \times \omega$, define the restriction $u \mid k \stackrel{\text { def }}{=}\{\langle n, m\rangle \mid u(n, m) \& u(m, k)\}$. It is straightforward to verify that

$$
\begin{aligned}
u \in T_{\xi} \Leftrightarrow & u \text { is a linear ordering of } \omega \\
& \& \exists k\left(u \mid k \notin W O^{<\xi+1}\right) \\
& \& \forall k\left[u \mid k \notin W O^{<\xi+1} \Rightarrow \exists k^{\prime}\left(u \mid k^{\prime} \notin W O^{<\xi+1} \& k^{\prime}<{ }_{u} k\right)\right] .
\end{aligned}
$$

Now, by induction, one can verify that: if $\nu<\omega_{1}$ is a limit ordinal (or zero) and $r<\omega$, then $W O^{<\nu}$ is $\Sigma_{\nu}^{0}$ and $W O^{<\nu+r}$ is $\Pi_{\nu+1}^{0} \cdot{ }^{2}$ In the R.H.S. above, the first clause is $\Pi_{1}^{0}$, the second clause is $\Sigma_{\xi+1}^{0}$, and the last clause is $\Pi_{\xi+2}^{0}$. This shows that $T_{\xi}$ is $\Pi_{\xi+2}^{0}$.

Now $A=F^{-1}\left[T_{\xi}\right]$, hence $A$ is $\Pi_{1+\xi+2}^{0}$. Unless $A$ is empty-a trivial case $-\xi>\omega$ and $1+\xi+2=\xi+2$.

The relativized version of this lemma should read: If $A$ is $\Sigma_{1}^{1}(y)$ and for all $x \in A, \omega_{1}^{(x, y)} \leq \xi$, then etc. Here $y \in Y$, an r.p. Polish space.

3.5. Theorem. For any $x \in S,[x]$ is $\Pi_{\omega_{1}^{x}+2}^{0}$.

Proof. Pick $\bar{x} \in[x]$ such that $\omega_{1}^{\bar{x}}=\omega_{1}^{[x]}=\xi$, say. Now set

$$
Z=\left\{y \in[\bar{x}] \mid \omega_{1}^{(y, \bar{x})}=\xi=\omega_{1}^{\bar{x}}\right\} .
$$

Clearly $Z$ is $\Sigma_{1}^{1}(\bar{x})$; we wish now to argue that:

(i) $Z$ is $\Pi_{\xi+2}^{0}$.

(ii) The transform: $Z^{*}=[\bar{x}]$.

(i) follows immediately from the relativized version of 3.4. As for (ii) note that, for $f \in G,\langle f \cdot \bar{x}, \bar{x}\rangle \in \Delta_{1}^{1}(f, \bar{x})$, hence $\omega_{1}^{(f \cdot \bar{x}, \bar{x})} \leq \omega_{1}^{(f, \bar{x})}$. It follows that $\{f \mid f \cdot \bar{x} \in Z\}=\left\{f \mid \omega_{1}^{(f \cdot \bar{x}, \bar{x})}=\omega_{1}^{\bar{x}}\right\} \supseteq\left\{f \mid \omega_{1}^{(f, \bar{x})}=\omega_{1}^{\bar{x}}\right\}$. Now this last set is comeager, by 3.1. Consequently, $\bar{x} \in Z^{*}$. Thus $Z^{*}$ is an invariant nonempty subset of $[\bar{x}]$. One can only have: $Z^{*}=[\bar{x}]$. $\Pi_{\xi+2}^{0}$.

Now, in view of (i) and Vaught's $1.4(\mathrm{iv})$, one gets that $Z^{*}$ (that is, $[\bar{x}]$ ) is

\footnotetext{
${ }^{2}$ This is far from optimal. See Stern's [33].
} 
3.6. Before proceeding to the main result of this section we need a few preliminaries. We define approximations $\mathscr{E}_{J}^{\nu}$ to $\mathscr{E}_{J}$, for $1 \leq \nu<\omega_{1}$, by

$$
x \mathscr{E}_{J}^{\nu} y \Leftrightarrow\left\{\begin{array}{l}
\text { for all } \Pi_{\nu}^{0} \text { invariant } A \subseteq S, \\
x \in A \Leftrightarrow y \in A .
\end{array}\right.
$$

Clearly the $\mathscr{E}_{J}^{\nu}$ form a decreasing sequence of equivalence relations, and because the orbits are Borel (cf. 1.3) one has

$$
\mathscr{E}_{J}=\bigcap_{1 \leq \nu<\omega} \mathscr{E}_{J}^{\nu}
$$

(These approximations are different from those defined by Burgess [2] for general analytic equivalence relations.)

3.7. Proposition. The relations $\mathscr{E}_{J}^{\nu}$ are $\Pi_{1}^{1}$.

Proof. We first wish to exhibit the uniformity in 1.4(iv) and (v). Let $U \subseteq \omega^{\omega} \times S$ be a $\Pi_{\nu}^{0}$ set whish is universal for $\Pi_{\nu}^{0}$ subsets of $S$. Define

$$
I: G \times \omega^{\omega} \times S \rightarrow \omega^{\omega} \times S
$$

by

$$
\langle g, \alpha, x\rangle \mapsto\langle\alpha, g \cdot x\rangle .
$$

$I$ is a $\Delta_{1}^{1}$ action of $G$ on $\omega^{\omega} \times S$. Set $T=U^{*}$, the ${ }^{*}$-transform relative to $I$. Then, for all $\alpha \in \omega^{\omega}$ and $x \in S,\langle\alpha, x\rangle \in T \Leftrightarrow\{g \mid\langle\alpha, g \cdot x\rangle \in U\}$ is comeager $\Leftrightarrow x \in\left(U_{\alpha}\right)^{*}$. So $T_{\alpha}=\left(U_{\alpha}\right)^{*}$.

We claim that $\left\{T_{\alpha} \mid \alpha \in \omega^{\omega}\right\}$ consists of, precisely, the $\Pi_{\nu}^{0}$ invariant subsets of $S$. Indeed, the equality above, together with 1.4(i) and 1.4(iv) show that $T_{\alpha}$ is $\Pi_{\nu}^{0}$ and $J$-invariant. Conversely, suppose $B \subseteq S$ is an invariant $\Pi_{\nu}^{0}$ set. Say $B=U_{\beta}$, for some $\beta \in \omega^{\omega}$, then again, $T_{\beta}=\left(U_{\beta}\right)^{*}=U_{\beta}$. This proves the claim.

We can thus write

$$
x \mathscr{C}_{J}^{\nu} y \Leftrightarrow \forall \alpha \in \omega^{\omega}\left(x \in T_{\alpha} \Leftrightarrow y \in T_{\alpha}\right) .
$$

$I$ being Borel measurable, it follows, using $1.4(\mathrm{v})$, that $T$ is Borel. Clearly now, the right-hand side of the equivalence is $\Pi_{1}^{1}$.

Remark. By using Louveau's separation theorem (see [14]), one can show that the $\mathscr{E}_{J}^{\nu}$ are actually Borel. We will not need this here.

3.8. Theorem. Suppose $A \subseteq S$ and $A$ is $\Sigma_{1}^{1}$ and invariant. If for all $x \in A$, $[x]$ is $\Pi_{\omega_{1}^{x}}^{0}$ then either $A / \mathscr{E}$ is countable or A meets perfectly many orbits.

Proof. To avoid needless technical clutter, we assume $J$ to be recursive, rather than $\Delta_{1}^{1}$. We shall explain at the end the modifications needed for the $\Delta_{1}^{1}$ case.

Assume towards a contradiction, that $A$ is a counterexample to our statement. Let $F: \omega^{\omega} \rightarrow A$ be a continuous surjection, and consider $\hat{\mathscr{E}}$, the pullback of $\mathscr{E}$ by $F$. $\hat{\mathscr{E}}$ is a $\Sigma_{1}^{1}$ equivalence relation with uncountably many, but not perfectly many, classes (cf. 1.6).

By a result of Stern [32, Theorem 3.2], under precisely these hypotheses, there is an invariant $\Sigma_{1}^{1}$ set, $Z^{\prime} \subseteq \omega^{\omega}$ with the following minimality property: $Z^{\prime}$ meets uncountably many $\widehat{\mathscr{E}}$-classes, but given any Borel invariant $C \subseteq \omega^{\omega}$ then one of the sets $C \cap Z^{\prime}$ or $Z^{\prime}-C$ meets only countably many classes. 
Let $Z=F\left[Z^{\prime}\right] . \quad Z$ is a $\Sigma_{1}^{1}$ subset of $A$, and one verifies easily that $Z$ enjoys in $S$ the same minimality property:

(*) $Z$ meets uncountably many orbits but given any Borel invariant $C \subseteq S$ then one of the sets $C \cap Z$ or $Z-C$ meets only countably many orbits.

Observe that by Silver's theorem, since $\mathscr{E}^{\nu}$ is a $\Pi_{1}^{1}$ equivalence relation, $Z / \mathscr{E}^{\nu}$ is countable. Indeed, since $Z$ does not meet perfectly many orbits, then a fortiori it does not meet perfectly many $\mathscr{E}^{\nu}$-classes.

We fix now $1 \leq \eta<\omega_{1}$ and let $\Sigma_{\eta}^{0}(Z)$ denote the collection of subsets of $Z$ which are $\Sigma_{\eta}^{0}$ in the space $Z$. Let us call an invariant set small if it meets only countably many orbits.

Claim 1. There is a largest small set in $\Sigma_{\eta}^{0}(Z)$. Indeed, if the contrary holds one gets a strictly increasing sequence $\left\langle B_{\xi} \mid \xi<\omega_{1}\right\rangle$ of invariant small $\Sigma_{\eta}^{0}(Z)$ sets. By 3.2 of [35], $B_{\xi}=\widetilde{B}_{\xi} \cap Z$, where $\widetilde{B}_{\xi}$ is invariant $\Sigma_{\eta}^{0}$. For $\xi<\omega_{1}$, suppose $x, y \in Z$, with $x \in B_{\xi}, y \notin B_{\xi}$; then actually $x \in \widetilde{B}_{\xi}$ and $y \notin \widetilde{B}_{\xi}$. Thus $x \mathscr{E}^{\eta} y$, showing that $Z / \mathscr{E}^{\eta}$ is uncountable-a contradiction. As usual, the axiom of choice could be dispensed with, here.

Now let $S_{\eta}$ denote the largest small set in $\Sigma_{\eta}^{0}(Z)$.

Claim 2. Suppose $x \in Z$ has its orbit in $\Pi_{\lambda}^{0}, \lambda$ limit, then $x \in \bigcup_{\xi<\lambda} S_{\xi}$. To prove this, let $[x]=\bigcap_{i<\omega} A_{i}$ where $A_{i}$ is in $\Sigma_{\xi_{i}}^{0}$, with $\xi_{i}<\lambda$. By passing to $A_{i}^{*}$ (and raising $\xi_{i}$ to $\xi_{i}+2$ ), one can assume that the $A_{i}$ 's are invariant.

Now, for each $i<\omega$, one of $Z \cap A_{i}$ or $Z-A_{i}$ is small (by the property $(*)$ of $Z$ ). If the second alternative were to hold for all $i<\omega$ then, since $Z-[x]=\bigcup_{i<\omega}\left(Z-A_{i}\right), Z-[x]$ would be a small set, and this is false. Thus, for some $r<\omega, Z \cap A_{r}$ is small, but then $[x] \subseteq Z \cap A_{r} \subseteq S_{\xi_{r}}$, and Claim 2 is proved.

$Z$ being $\Sigma_{1}^{1}$, say $Z$ is $\Sigma_{1}^{1}(\gamma)$ with $\gamma \in \omega^{\omega}$. Now define $F: \omega_{1} \rightarrow \omega_{1}$ by

$$
F(\xi)=\sup \left\{\omega_{1}^{[x, \gamma]} \mid x \in S_{\xi}\right\} .
$$

As usual, the set of almost fixed points of $F, K=\left\{\kappa<\omega_{1} \mid \forall \xi(\xi<\kappa \Rightarrow\right.$ $F(\xi)<\kappa)\}$, is closed and unbounded in $\omega_{1}$. Thus $K$ meets the set of $\gamma$ admissible ordinals $>\omega$. Let $\kappa \in K$ be such an ordinal.

By 3.3(b), $\operatorname{Spec}^{\gamma}(Z)$ is an intial segment of the $\gamma$-admissibles $>\omega$. Thus, either $\omega_{1}^{[x, \gamma]}<\kappa$ for all $x \in Z$, or else there is $u \in Z$ such that $\omega_{1}^{[u, \gamma]}=\kappa$. The latter alternative is impossible for, otherwise, since by hypothesis, $[u]$ is $\Pi_{\omega_{1}^{[u]}}^{0}$ and since $\omega_{1}^{[u]} \leq \omega_{1}^{[u, \gamma]}=\kappa$, one would have: $[u]$ is $\Pi_{\kappa}^{0}$ and hence by Claim 2, $[u]$ is in $S_{\xi}$ for some $\xi<\kappa$. But then by definition of $F$ we have $\omega_{1}^{[u, \gamma]}<\kappa$-a contradiction.

Thus we must have that for all $x \in Z, \omega_{1}^{[x, \gamma]}<\kappa$ (hence a fortiori $\omega_{1}^{[x]}<\kappa$ ). Consequently-using 3.5 or the hypothesis-for all such $x,[x]$ is in $\Pi_{\kappa}^{0}$. Thus $\mathscr{E}^{\kappa}|Z=\mathscr{E}| Z$. Now $Z / \mathscr{E}$ is uncountable whereas $Z / \mathscr{E}^{\kappa}$ is countable. This final contradiction concludes the proof.

As promised, we examine now the modifications needed for the case where $J$ is $\Delta_{1}^{1}$. Suppose that $J$ is $\Sigma_{1+\mu}^{0}$-measurable. (1) In the proof of Claim 1, we needed: $B_{\xi}=\widetilde{B}_{\xi} \cap Z$, where $\widetilde{B}_{\xi}$ is invariant $\boldsymbol{\Sigma}_{\eta}^{0}$. In $[35,3.2]$, this $\widetilde{B}_{\xi}$ is just $C_{\xi}^{\Delta}$ where $C_{\xi}$ is a $\Sigma_{\eta}^{0}$ subset of $S$ having $B_{\xi}=C_{\xi} \cap Z$. By $1.4(\mathrm{v}), C_{\xi}^{\Delta}$ 
is in $\Sigma_{\mu+\xi}^{0}$. Thus if $\xi \geq \mu^{2}$ then $\mu+\xi=\xi$ and $C_{\xi}^{\Delta}$ is still in $\Sigma_{\xi}^{0}$. (2) In the proof of Claim 2, passing from $A_{i}$ to $A_{i}^{*}$ we had to raise $\xi_{i}$ to $\xi_{i}+2$. With the present setup, $A_{i}^{*}$ is in $\Sigma_{\mu+\xi_{i}+2}^{0}$. Again, if $\lambda$ in this proof is assumed to be $\geq \mu^{2}$, then the argument goes through. (3) Finally, all that is needed to finish is to pick $\kappa \in K, \kappa \geq \mu^{2}$.

Remarks. (1) For the general case of Polish actions, Theorem 3.8 is, to my knowledge, the best known so far.

(2) For the special case of the model theory actions $\omega ! \times X_{L} \rightarrow X_{L}$, defined in $\S 1,3.8$ is due to Makkaï [15]. His proof uses specifically model theoretic techniques. Further, Makkai's [15, 1.2] shows in essence, that the hypothesis "for all $x \in S:[x]$ is $\Pi_{\omega_{1}^{x}}^{0}$ " need not imply that $\mathscr{E}$ is Borel so that results like 3.8 above cannot simply be reduced to Silver's theorem. (See also 4.5, Remark 3.)

(3) If one admits strong axioms of infinity, then the strongest statement in the general direction of 3.8 is Steel's theorem from [31]: Assume $\forall x$ ( $x^{\#}$ exists). Suppose $E$ is a $\Sigma_{1}^{1}$ equivalence relation on a recursively presented Polish (or analytic) space $X$ and for every $x$, the E-class of $x$ is $\Sigma_{\omega_{1}^{x}+2}^{0}$, then $X / E$ is countable or $E$ admits perfectly many classes.

This is actually stated for $P C_{\omega_{1} \omega}$ classes of models but the proof indicated there works in the broader context. I have shown in [29] that a statement weaker than Steel's theorem implies that $\aleph_{1}$ is inaccessible in $L$. Thus for general $\Sigma_{1}^{1}$ equivalence relations some large cardinal hypothesis is required and it might well be that the "sharps" are the optimal hypothesis here.

(4) Theorem 3.8 should be true with the weaker hypothesis " $[x]$ is $\Sigma_{\omega_{1}^{x}+2}^{0}$ " replacing our " $[x]$ is $\Pi_{\omega_{1}^{x}}^{0}$." Short of this, let us state without proof

3.9. Theorem. Assume that $G$ is (isomorphic to) a closed subgroup of $\omega$ ! and $J$ is recursive. Suppose $A \subseteq S$ is $\Sigma_{1}^{1}$ and invariant, if for all $x \in A,[x]$ is $\Sigma_{\omega_{1}^{x}+2}^{0}$ then the same conclusion as in 3.7 holds.

The proof here uses special properties of $\omega$ ! (as in $\S 4$ ) which have no analogue in the general case. It is a rather untoward combination of the techniques of $\S \S 3$ and 4 together with forcing over admissible sets and should not be committed to print, as is.

\section{Closed subgroups of $\omega$ !}

The permutation group $\omega$ ! has special topological properties which allow a finer analysis of its actions. We exploit these properties in the spirit of Vaught's [35] and Burgess' [4]. It seems that many topological properties of the spaces of models $\mathscr{X}_{L, F}$ are attributable to $\omega$ !.

Recall that $\omega$ ! is a $G_{\delta}$ subset of the Polish space $\omega^{\omega}$. Thus $\omega$ !, with the induced topology, is a Polish space. (A natural complete metric-and a recursive presentation-can actually be put on $\omega$ !. We will not need these here.) For any finite bijection $s$ with domain and range in $\omega$ let:

$$
V_{s}=\{f \in \omega ! \mid f \supseteq s\} \text {. }
$$

The collection $\mathscr{B}$ of the $V_{s}$ forms a basis for the topology of $\omega$ ! with special properties. More generally, let $X \subseteq \omega$ ! be any subspace, and let $\mathscr{B}_{X}$ denote 
the induced basis. The reader can easily check the following property:

(P): Given $U \in \mathscr{B}_{X}$ and $f \in X$, then $U f \in \mathscr{B}_{X}$, and there is a neighborhood $V$ of $f$ such that for any $g \in V, U g=U f$.

For the remainder of this section we assume $G$ is a closed subgroup of $\omega$ !, and $J: G \times S \rightarrow S$ is a continuous action of $G$ on the Polish space $S$. This will be called a logic-type action.

We first introduce a technical notion. ${ }^{3}$

4.1. Definition. Given a topological space $X$, a family $\mathscr{F}$ of subsets of $X$ is called a Borel family of rank $\nu$ (here $1 \leq \nu<\omega_{1}$ ), if there is a decomposition

$$
\mathscr{F}=\left(\bigcup_{1 \leq \xi<\nu} P_{\xi}\right) \cup\left(\bigcup_{1 \leq \xi<\nu} S_{\xi}\right)
$$

such that: (i) $S_{1}$ consists of open sets. (ii) $P_{\xi}=\left\{X-A \mid A \in S_{\xi}\right\}$ for $1 \leq \xi<\nu$. (iii) Any $A \in S_{\xi}$, for $\xi>1$, is expressible as $A=\bigcup_{i<\omega} B_{i}$ with $B_{i} \in \bigcup_{\eta<\xi} P_{\eta}$. $\omega_{1}$

Clearly, in the situation above, one has $S_{\xi} \in \Sigma_{\xi}^{0}$ and $P_{\xi} \in \Pi_{\xi}^{0}$, for $1 \leq \xi<$

For $\mathscr{U}$ a collection of subsets of $X$, let $\langle\mathscr{U}\rangle$ denote the topology generated by $\mathscr{U}$. $\langle\mathscr{U}\rangle$ consists of all arbitrary unions of finite intersections of sets from $\mathscr{U}$. Our next lemma is well known under different guises (see $[18,2.2 ; 13$, Chapter III, §37]).

4.2. Lemma. Suppose $X$ is a Polish space with topology $\mathscr{T}$, and $\mathscr{F}$ is a countable Borel family of rank $\nu$. Then $\langle\mathscr{T} \cup \mathscr{F}\rangle$ is a Polish topology on $X$ whose open sets are $\Sigma_{\xi}^{0}$ (relative to $\mathscr{T}$ ).

Proof. The following is a quite standard fact: $(*)$ Suppose $\left\{\mathscr{T}_{i} \mid i<\omega\right\}$ is a countable collection of Polish topologies on the set $Y$ with the property that $\bigcap_{i<\omega} \mathscr{T}_{i}$ is Hausdorff. The topology $\mathscr{U}=\left\langle\bigcup_{i<\omega} \mathscr{T}_{i}\right\rangle$ is Polish.

To prove this, set $\mathbf{P}=\prod_{i<\omega}\left\langle Y, \mathscr{T}_{i}\right\rangle$. Check first that $\langle Y, \mathscr{U}\rangle$ is homeomorphic to the diagonal $D$, of $\mathbf{P}$. The fact that $\bigcap_{i<\omega} \mathscr{T}_{i}$ is Hausdorff is used, next, to verify that $D$ is closed in $\mathbf{P}$. Being a countable product of Polish spaces, $\mathbf{P}$ is Polish, and $D$ is a Polish subspace of $\mathbf{P}$.

We proceed now to prove the lemma, by induction on $\nu$. If

$$
\mathscr{F}=\left(\bigcup_{1 \leq \xi<\nu} P_{\xi}\right) \cup\left(\bigcup_{1 \leq \xi<\nu} S_{\xi}\right)
$$

as in 4.1 , and $1 \leq \mu<\nu$, let

$$
\mathscr{F}_{\mu}=\left(\bigcup_{1 \leq \xi<\mu} P_{\xi}\right) \cup\left(\bigcup_{1 \leq \xi<\mu} S_{\xi}\right)
$$

and set $\mathscr{U}_{\mu}=\left\langle\mathscr{T} \cup \mathscr{F}_{\mu}\right\rangle$. Each $\mathscr{U}_{\mu}$ is a Borel family of rank $\mu$.

The case $\nu=1$ is trivial, as then $\mathscr{F}=\varnothing$.

If $\nu=\mu+1, \mu \geq 1$, then by the induction hypothesis $\left\langle X, \mathscr{U}_{\mu}\right\rangle$ is a Polish space. By using clause (iii) of 4.1, each member of $S_{\mu}$ is seen to be a countable union of members of $\mathscr{U}_{\mu}$. Hence $S_{\mu}$ is a countable collection of $\mathscr{U}_{\mu}$-open

\footnotetext{
${ }^{3}$ In [25] we used a more restrictive definition which is not adequate for the proof of 4.3 below.
} 
sets. By clause (ii) now, $P_{\mu}$ is a countable collection of $\mathscr{U}_{\mu}$-closed sets; say $P_{\mu}=\left\{C_{i} \mid i<\omega\right\}$.

One checks easily that

$$
\mathscr{U}_{\nu}=\mathscr{U}_{\mu+1}=\left\langle\mathscr{U}_{\mu} \cup P_{\mu}\right\rangle=\left\langle\bigcup_{i<\omega}\left\langle\mathscr{U}_{\mu} \cup\left\{C_{i}\right\}\right\rangle\right\rangle .
$$

Recall that the topology generated by a closed set over a Polish topology is Polish. Thus, each $\left\langle\mathscr{U}_{\mu} \cup\left\{C_{i}\right\}\right\rangle$ is Polish. Setting $\mathscr{T}_{i}=\left\langle\mathscr{U}_{\mu} \cup\left\{C_{i}\right\}\right\rangle$ in (*) above, one gets that $\mathscr{U}_{\nu}$ is Polish.

For $\nu$ a limit ordinal, one checks easily that $\mathscr{U}_{\nu}=\left\langle\bigcup_{1 \leq \xi<\nu} \mathscr{U}_{\xi}\right\rangle$. By the induction hypothesis each $\mathscr{U}_{\xi}$, for $1 \leq \xi<\nu$, is a Polish topology. Thus another use of $(*)$ yields that $\mathscr{U}_{\nu}$ is Polish. This finishes the proof.

Remark. One can now give a proof of Proposition 1.1, by observing that the generating family $\left\{\operatorname{Mod}(\varphi, \vec{s}) \mid \varphi \in F, \vec{s} \in \omega^{<\omega}\right\}$, for the topology $\mathscr{T}_{F}$, is a countable Borel family (of rank some $\nu<\omega_{1}$ ), and then quoting 4.2.

We now use this result to provs a very useful Transfer Lemma, a finer version of a result of Burgess [4, Theorem 8].

4.3. Transfer Lemma. Suppose $J: G \times S \rightarrow S$ is a logic-type action and $A \subseteq S$ is $\Pi_{\nu+1}^{0}$ and invariant (with $1 \leq \nu<\omega_{1}$ ). There is a Polish topology $\mathscr{U}$ on $S$ such that:

(i) $\mathscr{U}$ is finer than the topology of $S$ and the $\mathscr{U}$-open sets are $\Sigma_{\nu}^{0}$.

(ii) $A$ is $G_{\delta}$ relative to $\mathscr{U}$. $\mathscr{U})$.

(iii) $J: G \times S_{\mathscr{U}} \rightarrow S_{\mathscr{U}}$ is continuous (here $S_{\mathscr{U}}$ is $S$ equipped with the topology

Proof. Suppose $A=\bigcap_{i<\omega} B_{i}$, where $B_{i} \in \Sigma_{\nu}^{0}$ and $B_{i}=\bigcup_{j<\omega} C_{i j}$, with $C_{i j} \in$ $\bigcup_{\xi<\nu} \Pi_{\xi}^{0}$. It is readily verified that there is a countable Borel family $\mathscr{F}$ of rank $\nu$, such that $\left\{C_{i j} \mid i, j<\omega\right\} \subseteq \mathscr{F}$. Suppose, as in Definition 4.1,

$$
\mathscr{F}=\left(\bigcup_{1 \leq \xi<\nu} P_{\xi}\right) \cup\left(\bigcup_{1 \leq \xi<\nu} S_{\xi}\right) .
$$

Now let, $\mathscr{B}$ be a countable basis for $G$ with the property $(\mathrm{P})$ and let here $U, V$ range over $\mathscr{B}$. Set

$$
\begin{aligned}
& \widetilde{P}_{\xi}=\left\{C^{* U} \mid C \in P_{\xi}, U \in \mathscr{B}\right\}, \\
& \widetilde{S}_{\xi}=\left\{C^{\Delta U} \mid C \in S_{\xi}, U \in \mathscr{B}\right\}, \\
& \widetilde{\mathscr{F}}=\left(\bigcup_{1 \leq \xi<\nu} \widetilde{P}_{\xi}\right) \cup\left(\bigcup_{1 \leq \xi<\nu} \widetilde{S}_{\xi}\right) .
\end{aligned}
$$

We claim that $\widetilde{F}$ is a Borel family of rank $\nu$. Indeed, Theorem 1.4 easily yields the three clauses of Definition 4.1 , for $\widetilde{F}$. One checks the clauses in succession. (i) $\widetilde{S}_{1}$ consists of open sets (by $1.4(\mathrm{iv})$ ). (ii) Let $C^{* U} \in \widetilde{P}_{\xi}$, with $C \in P_{\xi}$, then $\sim\left(C^{* U}\right)=(\sim C)^{\Delta U} \in \widetilde{S}_{\xi}$. Similarly, one shows that $D \in \widetilde{S}_{\xi}$ implies $\sim D \in \widetilde{P}_{\xi}$. (iii) Let $C^{\Delta U} \in \widetilde{S}_{\xi}$ with $C \in S_{\xi}, \xi>1$. Say $C=\bigcup_{i<\omega} D_{i}$ 
with $D_{i} \in \bigcup_{\eta<\xi} P_{\eta}$. One gets

$$
C^{\Delta U}=\bigcup_{i<\omega} D_{i}^{\Delta U}=\bigcup_{i<\omega} \bigcup_{V \subseteq U} D_{i}^{* V}
$$

Thus $C^{\Delta U}$ is a countable union of members of $\bigcup_{\eta<\xi} \widetilde{P}_{\eta}$, as required.

Clearly $\widetilde{\mathscr{F}}$ is countable. Thus, setting $\mathscr{U}=\langle\mathscr{T} \cup \widetilde{\mathscr{F}}\rangle$, where $\mathscr{T}$ is the topology of $S$, Lemma 4.2 yields that $\mathscr{U}$ is Polish. (i) in the present proposition is now easily checked. To prove (ii), observe that, $A$ being invariant:

$$
\begin{aligned}
A & =A^{*}=\bigcap_{i<\omega} B_{i}^{*}=\bigcap_{i<\omega U} \bigcap_{i}^{\Delta U} \\
& =\bigcap_{i<\omega} \bigcap_{U}\left(\bigcup_{j<\omega} C_{i j}\right)^{\Delta U}=\bigcap_{i<\omega} \bigcap_{U} \bigcup_{j<\omega} C_{i j}^{\Delta U} .
\end{aligned}
$$

Now each $C_{i j}^{\Delta U}$, being in $\widetilde{\mathscr{F}}$, is $\mathscr{U}$-open. Thus, $A$ is $G_{\delta}$ relative to $\mathscr{U}$.

Finally to check (iii), it is enough to verify that, for any $D \in \widetilde{F}, J^{-1}[D]$ is open in $G \times S_{\mathscr{U}}$. So suppose $D=C^{* U}$ with $C \in P_{\xi}, U \in \mathscr{B}$, and consider an arbitrary point $\left\langle f_{0}, x_{0}\right\rangle \in J^{-1}\left[C^{* U}\right]$. One checks that: for all $\langle g, y\rangle \in G \times S_{\mathscr{U}}$;

$$
g \cdot y \in C^{* U} \text { iff } y \in C^{* U g} \text {. }
$$

(See $[35,2.1]$.) Thus $x_{0} \in C^{* U f_{0}}$. Set $U_{0}=U f_{0}$, and use property $(\mathbf{P})$ of the basis $\mathscr{B}$. Namely, $U_{0} \in \mathscr{B}$, and there is $V \in \mathscr{B}$ an open neighborhood of $f_{0}$, such that $U g=U_{0}$, for all $g \in V \cdot V \times C^{* U_{0}}$ is open in $G \times S_{\mathscr{U}}$, and contains $\left\langle f_{0}, x_{0}\right\rangle$. Now, whenever $\langle g, y\rangle \in V \times C^{* U_{0}}, y \in C^{* U_{0}}=C^{* U g}$; hence $g \cdot y \in C^{* U}$, that is $\langle g, y\rangle \in J^{-1}\left[C^{* U}\right]$. We have just shown that $J^{-1}\left[C^{* U}\right]$ includes $V \times C^{* U_{0}}$, an open neighborhood of $\left\langle f_{0}, x_{0}\right\rangle$. This shows that $J^{-1}\left[C^{* U}\right]$ is open. $J^{-1}[D]$, for $D \in \widetilde{S}_{\xi}$, is similarily treated.

Our next proposition while very simple to prove has clear connections with model theory, this is discussed in 5.4 (see also Suzuki [34]). We state it in some generality.

4.4. Proposition. Let $J: H \times X \rightarrow X$ be a continuous action of the Polish group $H$ on the metrizable space $X$. Any nonmeager orbit $\mathscr{O} \subseteq X$ is $G_{\delta}$.

Proof. Since $\mathscr{O}$ has the Baire property one can write $\mathscr{O}=D \cup T$ with $D$ a nonmeager $G_{\delta}$ set, $T$ a meager set. We check first that $\mathscr{O}=D^{*} \cup T^{\Delta}$. From the definitions $D^{*} \subseteq \mathscr{O}^{*}=\mathscr{O}$ and $T^{\Delta} \subseteq \mathscr{O}^{\Delta}=\mathscr{O}$. For the converse inclusion, let $x \in \mathscr{O}$, if $x \notin T^{\Delta}$ then $\{g \mid g \cdot x \in T\}$ is meager. Thus $\{g \mid g \cdot x \in D\}$ is comeager, that is $x \in D^{*}$.

Now, in [5, 2.4], it is shown that: $T$ is meager implies $T^{\Delta}$ is meager. Thus $T^{\Delta} \varsubsetneqq \mathscr{O}$. $\mathscr{O}$ being an orbit and $T^{\Delta}$ being invariant: $T^{\Delta}=\varnothing$ and $\mathscr{O}=D^{*}$. Using now 1.4(iv), $D^{*}$ is $G_{\delta}$.

The main result of this section can be viewed as a sort of basis result for "thin" invariant sets. It generalizes and extends model-theoretic theorems about atomic models (see 5.4, below). 
4.5. Theorem. Suppose $J: G \times S \rightarrow S$ is a logic-type action and $\varnothing \neq A \subseteq S$ is invariant, without perfectly many orbits. For $1 \leq \nu<\omega_{1}$,

(a) If $A$ is $\Pi_{\nu+1}^{0}$ then $A$ contains a $\boldsymbol{\Pi}_{\nu+1}^{0}$ orbit.

(b) If $A$ is $\Sigma_{\nu+2}^{0}$ then $A$ contains a $\Sigma_{\nu+2}^{0}$ orbit.

Proof. (a) Let $\nu=1$. Then $A$ is $G_{\delta}$ and is thus a Polish subspace of $S$. Now restrict $J$, namely let $J^{\prime}=J \mid(G \times A)$ and consider $\mathscr{E}_{J^{\prime}} \cdot \mathscr{E}_{J^{\prime}}$, being analytic, has the property of Baire, in $A \times A$.

A well-known theorem in the subject ([20], see [32, 1.2], for a proof) says that: (*) An equivalence relation on a Polish space, which has the Baire property, either is nonmeager or admits perfectly many classes.

The second alternative is contrary to hypothesis. Thus $\mathscr{E}_{J^{\prime}}$ is nonmeager in $A \times A$. Thus, by the Ulam-Kuratowski theorem (see [22]), at least one of its sections is nonmeager in $A$. In other words, there is a nonmeager orbit, $\mathscr{O} \subseteq A$. Apply now 4.4 to the action $J^{\prime}: G \times A \rightarrow A$ to get $\mathscr{O}$ is $G_{\delta}$ in $A$, hence in $S$.

Now for $\nu>1$, the Transfer Lemma will bring us back to the case just proved. For $\varnothing \neq A \subseteq S$, an ir:variant $\boldsymbol{\Pi}_{\nu+1}^{0}$ set, let $\mathscr{U}$ be a topology on $S$ with properties as in the statement of 4.3. $A$ is $\Pi_{2}^{0}$ in $S_{\mathscr{U}}$, note also that the fact that $A$ does not have perfectly many orbits in the space $S$ is preserved when passing to $S_{\mathscr{U}}$. (Indeed, a perfect set of points in $S_{\mathscr{U}}$ contains a perfect compact set of points in $S_{\mathscr{U}}$ which is compact and perfect in $S$.) Using now the case $\nu=1$, one finds an orbit $\mathscr{O} \subseteq A$ which is $G_{\delta}$ is $S_{\mathscr{U}}$. Since each

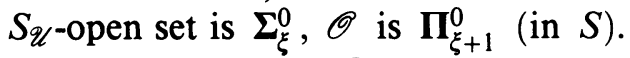

To prove (b), let $\mathscr{B}$ be a countable basis for $G$ with property (P). Set

$$
\widetilde{S}=S \times \mathscr{B},
$$

$\mathscr{B}$ being viewed as a countable discrete space, $\tilde{S}$ is a Polish space with the product topology. Let $\pi: \widetilde{S} \rightarrow S$ be the projection map: $\pi(x, U)=x$. Define

$$
\tilde{J}: G \times \tilde{S} \rightarrow \tilde{S}, \quad\langle g,\langle x, U\rangle\rangle \mapsto\left\langle g \cdot x, U g^{-1}\right\rangle .
$$

$\tilde{J}$ is clearly continuous and a logic-type action. Much as in Vaught's [35, §3], we define a new transform. Given $C \subseteq S$,

$$
C^{(*)}=\left\{\langle x, U\rangle \in \tilde{S} \mid x \in C^{* U}\right\} .
$$

Recall that: $x \in C^{* U}$ if and only if $g \cdot x \in C^{* U g^{-1}}$. This says: $\langle x, U\rangle \in C^{(*)}$ iff $\widetilde{J}(g,\langle x, U\rangle) \in C^{(*)}$. Consequently, $C^{(*)}$ is invariant in $\widetilde{S}$.

For $\eta \geq 1$, we claim that if $C \subseteq S$ is $\Pi_{\eta}^{0}$ then $C^{(*)}$ is $\Pi_{\eta}^{0}$ in $\tilde{S}$. In fact this is just the uniform version of $1.4(\mathrm{iv})$. (Alternatively: Set $D_{U}=G \times\{U\}$. $\left\{D_{U} \mid U \in \mathscr{B}\right\}$ is a partition of $\widetilde{S}$ into clopen sets. For each $U, C^{(*)} \cap D_{U}=$ $C^{* U} \times\{U\}$ which is a $\Pi_{\eta}^{0}$ set, by $1.4(\mathrm{iv})$. From here on, it is easy to verify that $C^{(*)}$ is $\boldsymbol{\Pi}_{\eta}^{0}$.)

Let now $\varnothing \neq A \subseteq S$ be invariant $\Sigma_{\nu+2}^{0}$. Say, $A=\bigcup_{i<\omega} C_{i}$, with $C_{i}$ in $\Pi_{\nu+1}^{0}$, then $A=A^{\Delta}=\bigcup_{i<\omega} C_{i}^{\Delta}$. Pick $r<\omega$ such that $C_{r}^{\Delta} \neq \varnothing$, then by 1.4(iii):

$$
C_{r}^{\Delta}=\bigcup_{U \in \mathscr{B}} C_{r}^{* U}=\pi\left[C_{r}^{(*)}\right] .
$$


Claim. $C_{r}^{(*)}$ does not contain perfectly maily $\tilde{J}$-orbits.

Assuming the contrary, let $P \subseteq C_{r}^{(*)}$ be a perfect compact set consisting of pairwise $\mathscr{E}_{\widetilde{J}}$ inequivalent elerients. Set $Q=\pi[P], Q$ is a subset of $C_{r}^{\Delta} \subseteq A$, and is perfect in $S$. Let $\mathscr{E}^{\prime}=\mathscr{E}_{J} \cap(Q \times Q)$. Observe now that the image under $\pi$ of a $\widetilde{J}$-orbit is a $J$-orbit, and that at most countably many $\widetilde{J}$-orbits are mapped to the same $J$-orbit. Consequently, the $\mathscr{E}^{\prime}$-classes in $Q$ are all countable, hence meager. Since $\mathscr{E}^{\prime}$ has the Baire property in $Q \times Q$, we can apply the Ulam-Kuratowski theorem and conclude that $\mathscr{E}^{\prime}$ is meager. Now by the proposition $(*)$ at the beginning of this proof, $\mathscr{E}^{\prime}$ has perfectly many classes. Hence $A$ contains perfectly many $\mathscr{E}_{J}$-orbits. This contradiction proves the claim.

Now since $C_{r}^{(*)}$ is $\Pi_{\nu+1}^{0}$ in $\widetilde{S}$, one can apply (a) above to this set. Let thus $\tilde{\mathscr{O}} \subseteq C_{r}^{(*)}$ be a $\Pi_{\nu+1}^{0} \tilde{J}$-orbit. Clearly, $\pi[\tilde{\mathscr{O}}]$ is a $J$-orbit included in $A$. Further, since $\pi[\tilde{\mathscr{O}}]=\bigcup_{U \in \mathscr{B}}\left\{x \in S \mid\langle x, U\rangle \in C_{r}^{(*)}\right\}$, hence $\pi[\widetilde{\mathscr{O}}]$ is a countable union of $\boldsymbol{\Pi}_{\nu+1}^{0}$ sets. This concludes the proof of (b).

Remarks. (1) It is clear that the proof of the case $\Pi_{2}^{0}$ in (a) above does not need special properties of $G$. It works in the general context of a continuous action of a Polish group on a Polish space.

(2) In contradistinction to our work in $\S 2$, essential use is made here of the fact that the space $S$ is Polish (rather than, say, analytic). Theorem 4.5 cannot extend to the analytic case.

(3) 4.6(a) together with Louveau's theorem [14], were used in [28] to show that the relation: $R(x, u) \Leftrightarrow$ " $x \in S \& u \in W O \&[x] \in \Pi_{|u|}^{0}$ " is $\Pi_{1}^{1}$. An easy consequence of this is that, for a recursive logic-type action, the hypothesis: for all $x \in S$ : Borel rank $([x])<\omega_{1}^{x}$, implies that $\mathscr{E}_{J}$ is $\Delta_{1}^{1}$.

We now apply 4.5 to a situation rather different from what has been considered so far.

4.6. Theorem. Let $J: G \times S \rightarrow S$ be a logic-type action. If the number of orbits is finite, say $n$, then for $n$ odd [resp. even] all the orbits are in $\Sigma_{p}^{0} \cup \Pi_{p}^{0}$ [resp. $\left.\Delta_{p}^{0}\right]$, where $p=\left[\frac{n}{2}\right]+2$.

Proof. One simply applies 4.5 repeatedly. By $4.5(\mathrm{a})$, let $\mathscr{O}_{1} \subseteq S$ be a $\Pi_{2}^{0}$ orbit. $S-\mathscr{O}_{1}$ is $\Sigma_{2}^{0}$ (hence $\Sigma_{3}^{0}$ ). If it is nonempty then, by $4.5(\mathrm{~b})$ a $\Sigma_{3}^{0}$ orbit $\mathscr{O}_{2} \subseteq S-\mathscr{O}_{1}$ can be found. Consider next the $\Pi_{3}^{0}$ set $S-\left(\mathscr{O}_{1} \cup \mathscr{O}_{2}\right)$ etc. It is easily verified that, when the process terminates, the orbits $\mathscr{O}_{1}, \ldots, \mathscr{O}_{n}$ are indeed in $\Sigma_{p}^{0} \cup \boldsymbol{\Pi}_{p}^{0}$ for $n$ odd, and in $\Delta_{p}^{0}$ for $n$ even.

Let us observe finally that 4.5 yields a "picture" of what a counterexample (if any) to the T.V.C. in a logic-type action $J: G \times S \rightarrow S$ would look like. Indeed, in such a situation, for each $\nu \geq 1$, since $S / \mathscr{E}^{\nu+1}$ is countable, the number of $\Sigma_{\nu+1}^{0}$ orbits must be countable. Their union $T_{\nu+1}$ is a $\Sigma_{\nu+1}^{0}$ set. Since $S-T_{\nu+1}$ is nonempty, then by $4.5(\mathrm{~b})$, one can find an orbit $\mathscr{O}_{\nu} \subseteq S-T_{\nu+1}$. $\mathscr{O}_{\nu}$ is in $\Pi_{\nu+1}^{0}-\Sigma_{\nu+1}^{0}$. Thus the Borel ranks of the orbits would have to be densely packed in this sense.

\section{MODEL THEORY}

5.1. In this last section, we briefly discuss the connection between the descriptive set theoretic notions used in this paper and model theoretic notions. 
Arguments will just be sketched, as they consist mostly of routine verifications.

For the purposes of this discussion, let $L$ be a recursive first-order language with only relation and constant symbols. The set $X_{L}$ of $L$-structures with universe $\omega$ is essentially a countable product of sets $\mathscr{P}\left(\omega^{n}\right)$ and $\omega^{k}$. In some of the arguments below, we pass from $X_{L}$ to $X_{L} \times \omega^{n}$, implicitly augmenting the language with $n$ new constant symbols. $F$ shall denote a countable fragment of $L_{\omega_{1} \omega}$. Recall that $F$ need only contain the atomic formulas and be closed under negation and subformulas. The more usual fragments, closed as well under $\exists, \forall$ and boolean combinations will be called standard fragments. Recall the notation of $\S 1, \mathscr{X}_{L, F}=\left\langle X_{L}, \mathscr{T}_{F}\right\rangle$. We shall drop $L$ from here on and write $\mathscr{X}_{F}$. For the smallest fragment $F_{0}$ (consisting of the atomic formulas and their negations), just write $\mathscr{X} . \mathscr{X}$ is homeomorphic to the Cantor space. $\vec{x}$ here stands ambigiously for $x_{1}, \ldots, x_{n}$ and the notation $\varphi(\vec{x})$ is meant to imply that $\varphi$ has its free variables among $\vec{x}$. For $\varphi(\vec{x}) \in L_{\omega_{1} \omega}$, set $\operatorname{Mod}^{n}(\varphi)=$ $\left\{\left(\dot{r^{\prime}}, \vec{t}\right) \in \mathscr{a}^{\mathfrak{O}} \times \omega^{n} \mid \mathcal{N} \models \varphi[\vec{t}]\right\}$.

5.2. As in Vaught's [35] and Miller's [17], one can define the Borel hierarchy of formulas relative to $F$.

$$
\begin{aligned}
& F-\Sigma_{0}^{\prime 0}=\{\varphi \mid \varphi \text { is a finite conjunction of formulas of } F\}, \\
& F-\Pi_{\xi}^{\prime 0}=\left\{\neg \varphi \mid \varphi \in F-\Sigma_{\xi}^{\prime 0}\right\},
\end{aligned}
$$

where for $1 \leq \xi \leq \omega_{1}$,

$F-\Sigma_{\xi}^{\prime 0}=$ The collection of $\varphi \in L_{\omega_{1} \omega}$ of the form:

$$
\varphi(\vec{y})=\bigvee_{k<\omega} \exists x_{1} \cdots x_{k} \theta_{k}\left(x_{1}, \ldots, x_{k}, \vec{y}\right),
$$

where $\theta_{k}$ is in $\bigcup_{\zeta<\xi} F-\Pi_{\zeta}^{\prime 0}$. For $F=F_{0}$, we drop the prefix $F_{0}$. We shall often say that a formula belongs to one of these classes when one of its trivial logical variants belongs to that class.

The following is an inessential extension, to fragments other than $F_{0}$, of Vaught's [35, 3.2]. (See [17, §3], for standard fragments.) Vaught's proof works, unchanged, in our setting.

Theorem. The invariant $\Sigma_{\xi}^{0}$ sets in the space $\mathscr{X}_{F}$ coincide with the sets of the form $\operatorname{Mod}^{0}(\varphi)$ where $\varphi$ is a $F-\Sigma_{\xi}^{\prime 0}$ sentence.

5.3. Our next remarks are meant to relate the hypotheses of Theorems 3.8 and 3.9 to more orthodox model theoretic-notions. Given an $L$-structure $\mathscr{M} \in \mathscr{X}$, let HYP $_{\mathscr{M}}$ denote, as in Barwise [1, Chapter II], the smallest admissible over $\mathscr{M} . O(\mathscr{M})$ is the ordinal height of $\mathrm{HYP}_{\mathscr{M}}$. The reader familiar with [1] can easily verify that, except for the case $O(\mathscr{M})=\omega$, one has $\omega_{1}^{[\mathscr{M}]}=O(\mathscr{M})$. Let here $\mathbf{F}_{\mathscr{M}}$ denote the (standard) fragment HYP $_{\mathscr{M}} \cap L_{\omega_{1} \omega}$, and $\operatorname{Th}(\mathscr{M})$ the set of sentences of $\mathbf{F}_{\mathscr{M}}$ true in $\mathscr{M}$.

We assume now familiarity with [1, Chapter VII], or Nadel's [21]. For $\varphi \in$ $L_{\omega_{1} \omega}, \operatorname{qr}(\varphi)$ denotes the quantifier rank of $\varphi[1$, p. 296]. It is easily verified, by induction, that for $\varphi \in \bigcup_{\zeta} \Sigma_{\zeta}^{\prime 0}, \varphi \in \Sigma_{\xi}^{\prime 0} \Leftrightarrow \operatorname{qr}(\varphi) \leq \xi \cdot \omega$. Given a model $\mathscr{M}$ and $\vec{t} \in \omega^{n}, \sigma_{\mathscr{M}, \vec{t}}^{\xi}$ is the $\xi$-characteristic of $\vec{t}$ in $\mathscr{M}$ as defined in [1, p. 298]. One should keep in mind that if $\xi<O(\mathscr{M})$, then $\sigma_{\mathscr{M}, \vec{t}}^{\xi} \in \mathrm{HYP}_{\mathscr{M}}$, thus it belongs to the fragment $\mathbf{F}_{\mathscr{M}}[1, \mathrm{VII}, 7.1]$. 
Proposition. Assume $O(\mathscr{M})>\omega$ and set $\mu=\omega_{1}^{[\mathscr{M}]}$. In the space $\mathscr{X}$,

(a) $[\mathscr{M}]$ is $\Pi_{\mu}^{0}$ if and only if the $\mathbf{F}_{\mathscr{M}}$-theory of $\mathscr{M}$ is $\aleph_{0}$-categorical.

(b) $[\mathscr{M}]$ is $\mathbf{\Pi}_{\mu+1}^{0}$ if and only if $\mathscr{M}$ is an $\mathbf{F}_{\mathscr{M}}$-atomic model.

(c) $[\mathscr{M}]$ is $\Sigma_{\mu+2}^{0}$ if and only if there is $\vec{s} \in \omega^{<\omega}$ such that $(\mathscr{M}, \vec{s})$ is an $\mathbf{F}_{(\mathscr{K}, \vec{s})}$-atomic model.

Proof. The implications from right to left are straightforward computations from the definitions. As for the converses:

(a) By applying 7.2 of [1, Chapter VII] to the admissible fragment $\mathbf{F}_{\mathscr{M}}$, if $\mathscr{N} \models \operatorname{Th}(\mathscr{M})$ then $\mathscr{M}$ and $\mathscr{N}$ satisfy the same sentences of $L_{\omega_{1} \omega}$ with quantifier rank $\leq \mu$. Using 5.2, this implies that $\mathscr{N}$ belongs to every $\Pi_{\mu}^{0}$ invariant subset of $\mathscr{X}$. Thus, if $[\mathscr{M}]$ is $\Pi_{\mu}^{0}$ then $[\mathscr{M}]=\operatorname{Mod}^{0}(\bigwedge \operatorname{Th}(\mathscr{M}))$, and $\operatorname{Th}(\mathscr{M})$ is $\aleph_{0}$-categorical.

(b) Suppose $[\mathscr{M}]$ is $\Pi_{\nu+1}^{0}$. We claim that $\mathscr{M}$ is in fact $\Pi_{2}^{0}$ in the space $\mathscr{X}_{\mathbf{F}_{\mathscr{K}}}$. Granting this, since $\mathbf{F}_{\mathscr{M}}$ is a standard fragment, Miller's [17, 3.1] says that in precisely this situation, $\mathscr{M}$ is $\mathbf{F}_{\mathscr{M}}$-atomic. Now, to prove the claim, use 5.2 to get $[\mathscr{M}]=\operatorname{Mod}^{0}(\psi)$, where $\psi=\bigwedge_{n<\omega} \forall x_{1} \cdots x_{n} \bigvee_{m<\omega} \varphi_{n m}(\vec{x})$ with each $\varphi_{n m}$ in $\bigcup_{\zeta<\mu} \Sigma_{\zeta}^{\prime 0}$. Given $n<\omega, \vec{t} \in \omega^{n}$, there is an $m<\omega$ such that $\mathscr{M} \vDash \varphi_{n m}[\vec{t}]$. Set $\theta_{\vec{t}}=\sigma_{\mathscr{M}, \vec{i}}^{\xi}$ where $\xi=\operatorname{qr}\left(\varphi_{n m}\right)$ and note that $\xi<\mu$. Thus, $\theta_{\vec{t}} \in \mathbf{F}_{\mathscr{M}}$. Now, using the principal result on the $\xi$-characteristics [1, VII, 6.3], the reader can easily verify that $[\mathscr{M}]=\operatorname{Mod}^{0}\left(\bigwedge_{n<\omega} \forall x_{1} \cdots x_{n} \bigvee_{\vec{t} \in \omega^{n}} \theta_{\vec{t}}(\vec{x})\right)$. A direct computation (or 5.2) now shows that $[\mathscr{M}]$ is indeed $\Pi_{2}^{0}$ in $\mathscr{X}_{\mathrm{F}_{\mathscr{K}}}$, as claimed.

(c) Using 5.2, one can get a $\varphi(\vec{x}) \in \Pi_{\nu+1}^{\prime 0}$ such that $\mathscr{M}=\operatorname{Mod}^{0}(\exists \vec{x} \varphi(\vec{x}))$. Observe that, in the space $\mathscr{X} \times \omega^{n}$, the set $A=\operatorname{Mod}^{n}(\varphi(\vec{x}))$ is an invariant $\Pi_{\mu+1}^{0}$ set consisting of at most countably many orbits, all of the form $[(\mathscr{M}, \vec{t})]$, where $\vec{t} \in \omega^{n}$. Apply now Theorem 4.5 (a) to $A$, to conclude that $A$ includes a $\boldsymbol{\Pi}_{\mu+1}^{0}$ orbit, say $[(\mathscr{M}, \vec{a})]$. Next, apply (b) of the present proposition to $(\mathscr{M}, \vec{a})$, in the space $\mathscr{X} \times \omega^{n}$.

5.4. From a certain perspective, Theorem 4.5 extends and generalizes a wellknown existence theorem for atomic models (namely: Theories with "few" models have atomic models). Indeed, recall the results from Suzuki [34] and Miller [17]: Given a countable standard fragment $F, \mathscr{M} \in \mathscr{X}$ is $F$-atomic, if and only if $[\mathscr{M}]$ is $\Pi_{2}^{0}$ in $\mathscr{X}_{F}$. Theorem $4.5(\mathrm{a})$, for the case $\nu=1$ asserts the existence of $\boldsymbol{\Pi}_{2}^{0}$ orbits under a wider variety of situations. Thus, even this special case gives new information as it allows one to deal with rather more general fragments than the standard ones, and thus produce analogues of the atomic models for these fragments. (Independently, Parigot [23] has studied model theoretic notions for more general types of fragments, not closed under negation. Without getting into details here, the topological spaces associated with Parigot's fragments are not Polish but can be shown to be Baire spaces, where every $G_{\delta}$ subspace is a Baire space.)

5.5. Theorems 4.5 and 4.6 (or variants thereof) can be used to derive apparently new model theoretic results-even for finitary first-order logic.

To illustrate the method, consider a consistent first-order theory $T$ with $\forall_{k}^{0}$ axioms $(k \geq 2)$. Let $F$ be the fragment $F=\exists_{k-2}^{0} \cup \forall_{k-2}^{0}$. The set of models 
of $T, A=\{\mathscr{M} \in \mathscr{X} \mid \mathscr{M} \vDash T\}$, is a $\Pi_{2}^{0}$ subset of $\mathscr{X}_{F}$. Under the assumption that $T$ has infinite models, one gets by applying 4.5(a) to $A$ :

(a) If $T$ has fewer than $2^{\aleph_{0}}$ countable models, then $T$ has a model $\mathscr{M}$ such that $[\mathscr{M}]$ is $\Pi_{2}^{0}$ in the space $\mathscr{X}_{F}$.

(b) Using 5.2 above, it is readily verified that $\mathscr{M}$ is characterized by a sentence

$$
\sigma=\bigwedge_{n<\omega} \forall x_{1} \cdots x_{n} \bigvee_{m<\omega} \varphi_{n m}(\vec{x})
$$

where the $\varphi_{n m}$ are in $\exists_{k-1}^{0} . \mathscr{M}$ is by necessity $L_{\omega \omega}$-atomic, since $[\mathscr{M}]$ is $\Pi_{2}^{0}$ in $\mathscr{X}_{L_{\omega \omega}}($ see $[17,3.1])$.

(c) If, further, $T$ has only finitely many countable models (say $n$ ), then by applying 4.6 followed by 5.2, one sees that the orbits of its models can be characterized by sentences in $\left(F-\Sigma_{p}^{\prime 0}\right) \cup\left(F-\Pi_{p}^{\prime 0}\right)$ [resp. $F-\Delta_{p}^{\prime 0}$ ] if $n$ is odd [resp. even], where $p=\left[\frac{n}{2}\right]+2$.

Computed relative to the Cantor topology (i.e., the space $\mathscr{X}$ ) this says that the orbits are in the Borel class $\Sigma_{q}^{0} \cup \Pi_{q}^{0}$ [or $\Delta_{q}^{0}$, as the case may be], where $q=\left[\frac{n}{2}\right]+k$.

Added in proof. In Borel Actions of Polish Groups [to appear], H. Becker and A. S. Kechris have recently established the equivalence of the T.V.C. with the more general conjecture: A Borel action of a Polish group on a standard Borel space has either countably many or prefectly many orbits. (See the discussion in $\S 1.2$, above.) They have also proved a generalization of the Transfer Lemma (4.3) to general Polish groups (albeit with coarser bounds).

\section{REFERENCES}

1. J. Barwise, Admissible sets and structures, Springer-Verlag, Berlin, 1975.

2. J. P. Burgess, A reflection phenomenon in descriptive set-theory, Fund. Math. 94 (1979), 127-139.

3. __ Equivalences generated by families of Borel sets, Proc. Amer. Math. Soc. 69 (1978), 323-326.

4. __ Two selection theorems, Bull. Greek Math. Soc. 18 (1977), 121-136.

5. J. P. Burgess and D. E. Miller, Remarks on invariant descriptive set theory, Fund. Math. 90 (1975), 53-75.

6. J. Calabrix and J. P. Troallic, Applications séparément continues, C.R. Acad. Sci. Paris Sér. A 288 (1975), 647-648.

7. C. C. Chang and H. J. Keisler, Model theory, North-Holland, Amsterdam, 1973.

8. J. Dixmier, Duel et quasi-duel d'une algèbre de Banach involutive, Trans. Amer. Math. Soc. 104 (1962), 278-283.

9. R. O. Gandy, On a problem of Kleene's, Bull. Amer. Math. Soc. 66 (1960), 501-502.

10. A. Grzegorczyk, A. Mostowski, and C. Ryll-Nardjewski, Definability of sets in models of axiomatic theories, Bull. Acad. Polon. Sci. Sér. Sci. Math. Astronom. Phys. 9 (1961), 163167.

11. P. G. Hinman, Some applications of forcing to hierarchy problems in arithmetic, Z. Math. Logik Grundlag. Math. 15 (1969), 341-352.

12. A. S. Kechris, Measure and category in effective descriptive set-theory, Ann. Math. Logic 5 (1973), 337-384.

13. K. Kuratowski, Topology, vol. I, Academic Press, New York, 1966-1968.

14. A. Louveau, A separation theorem for $\Sigma_{1}^{1}$ sets, Trans. Amer. Math. Soc. 260 (1980), 363378. 
15. M. Makkai, An example concerning Scott heights, J. Symbolic Logic 46 (1981), 301-318.

16. D. E. Miller, On the measurability of orbits in Borel actions, Proc. Amer. Math. Soc. 63 (1977), 165-170.

17 _ The invariant $\Pi_{\alpha}^{0}$ separation principle, Trans. Amer. Math. Soc. 242 (1978), 185-204.

18. _ Borel selectors for separated quotients, Pacific J. Math. 91 (1980).

19. Y. N. Moschovakis, Descriptive set theory, North-Holland, Amsterdam, 1980.

20. J. Mycielski, Independent sets in topological algebras, Fund. Math. 55 (1964), 139-147.

21. M. Nadel, Scott sentences and admissible sets, Ann. Math. Logic 7 (1974), 267-294.

22. J. C. Oxtoby, Measure and category, Springer-Verlag, Berlin, 1971.

23. M. Parigot, Omission des types dans les théories $\Pi_{2}^{0}(\Delta)$, C. R. Acad. Sci. Paris Sér. I 299 (1984), 195-198.

24. J. P. Ressayre, Models with compactness properties with respect to logics on admissible sets, Ann. Math. Logic 11 (1977), 31-55.

25. C. A. Rogers et al., Analytic sets, Academic Press, London, 1980.

26. C. Ryll-Nardjewski, On Borel measurability of orbits, Fund. Math. 56 (1984), 129-130.

27. G. E. Sacks, Countable admissible ordinals and hyperdegrees, Adv. in Math. 19 (1976), 213-262.

28. R. L. Sami, Sur la conjecture de Vaught en théorie descriptive, C. R. Acad. Sci. Paris Sér. I 295 (1982), 413-416.

$29 \ldots$, On $\Sigma_{1}^{1}$ equivalence relations with Borel classes of bounded rank, J. Symbolic Logic 49 (1984), 1273-1283.

30. J. Silver, Counting the number of classes of Borel and co-analytic equivalence relations, Ann. Math. Logic 18 (1980), 1-28.

31. J. R. Steel, On Vaught's conjecture, Cabal Seminar 1976-1977, Lecture Notes in Math., vol. 689, Springer-Verlag, Berlin, 1978.

32. J. Stern, Effective partitions of the real line into Borel sets of bounded rank, Ann. Math. Logic 18 (1980), 29-60.

33. _ـ Evaluation du rang de Borel de certains ensembles, C. R. Acad. Sci. Paris Sér. I 286 (1978), 855-857.

34. Y. Suzuki, Orbits of denumerable models of complete theories, Fund. Math. 67 (1970), 8995.

35. R. L. Vaught, Invariant sets in topology and logic, Fund. Math. 82 (1974), 269-293.

U.F.R. De Mathématiques, Université Paris ViI, 75251 Paris Cedex 05, France 